\title{
Searching for High Energy Neutrinos detected by ANTARES in coincidence with Gravitational Wave signals observed during LIGO Observation Run 01
}

\author{
Thierry Pradier*† \\ Université de Strasbourg, CNRS, IPHC UMR 7178, F-67000 Strasbourg, France \\ E-mail: thierry.pradier@iphc.cnrs.fr
}

\section{Bruny Baret}

APC, Université Paris Diderot, CNRS/IN2P3, CEA/IRFU, Observatoire de Paris, Sorbonne Paris Cité, 75205 Paris, France

E-mail: bruny .bareteapc.univ-paris7.fr

\author{
Alexis Coleiro \\ APC, Université Paris Diderot, CNRS/IN2P3, CEA/IRFU, Observatoire de Paris, Sorbonne \\ Paris Cité, 75205 Paris, France \\ now at IFIC - Instituto de Física Corpuscular (CSIC - Universitat de València) c/ Catedrático \\ José Beltrán, 2 E-46980 Paterna, Valencia, Spain \\ E-mail: alexis.coleiro@apc.univ-paris7.fr
}

\section{on behalf of the ANTARES Collaboration}

The ANTARES Neutrino Telescope can determine the arrival direction of a muonic High Energy Neutrino (HEN) with a precision well below $1^{\circ}$ above $1 \mathrm{TeV}$. The detection of such a HEN in coincidence with a Gravitational Wave (GW) event would then improve the localization of the GW source, facilitating the search for electromagnetic counterparts. The results of such targeted HEN searches for the $3 \mathrm{GW}$ events (GW150914, GW151226, both confirmed signals, and LVT151012, an event candidate) detected during the Observation Run O1 of ADVANCED LIGO in 2015-2016 are presented. The principles of a sub-treshold analysis, which looks for time and space correlations between HEN detected by ANTARES or ICECUBE and GW candidates of low signalto-noise ratio detected by LIGO during $\mathrm{O} 1$ are presented. The specific procedure optimized to select HEN candidates in ANTARES data is emphasized.

35th International Cosmic Ray Conference - ICRC2017

10-20 July, 2017

Bexco, Busan, Korea

\footnotetext{
*Speaker.

${ }^{\dagger}$ The GW+HEN analyses presented here are the results of a collaborative work between the ANTARES Collaboration, the LIGO Scientific Collaboration, the VIRGO Collaboration and the ICECUBE Collaboration. Thanks in particular to I. Bartos (Columbia University, New-York, USA) for LIGO/VIRGO, and C. Finley (Oskar Klein Centre and Dept. of Physics, Stockholm, Sweden), for ICECUBE.
} 


\section{Introduction}

The observation of three significant gravitational wave (GW) sources by ADVANCED LIGO on Sept. $14^{\text {th }}, 2015$, Dec. $26^{\text {th }}, 2015$ and Jan. $4^{\text {th }}, 2017[1,2,3]$ represents an important step forward in the study of the latest stage of massive star binary system evolution. By involving a number of observatories from the radio to the gamma-rays and also neutrino detectors to search for a potential counterpart associated to these events, the detection of the first GW events opened the era of time domain multi-messenger astrophysics.

The three signals correspond to the coalescence of stellar-mass black hole binary (BBH) systems at distances ranging from $400 \mathrm{Mpc}$ to about $900 \mathrm{Mpc}$. While an electromagnetic counterpart (presumably associated with a neutrino emission) is generally expected from a neutron star/black hole or neutron star/neutron star merger [4], current consensus is that black hole/black hole merger does not produce electromagnetic or neutrino counterpart. However, in a dense enough hadronic environment, an accretion disk might form, and relativistic outflow connected to the accretion could be released. Energy dissipation in this outflow would consequently lead to a gamma-ray emission with a potential high-energy neutrino (HEN, of energy $\gg \mathrm{GeV}$ ) counterpart [5]. The detection of HEN from GW sources would provide a better determination of the origin of the GW signal, thanks to the comparatively much more accurate pointing accuracy of neutrino telescopes, of the order of (or below) the degree - and would subsequently help the electromagnetic follow-up campaign.

ANTARES is currently the largest high-energy neutrino telescope in the Northern hemisphere [6]. Located in the Mediterranean Sea, $40 \mathrm{~km}$ off the Southern coast of France, it is composed of 12 detection lines that detect the Cherenkov light emitted by relativistic upgoing muons, signature of a neutrino interaction close to the detector. After the discovery from ICECUBE of a cosmic neutrino flux of still unknown origin [7], observing campaigns relying both on electromagnetic and multi-messenger facilities may be decisive to identify the origin of these neutrino events.

Joint searches of common sources of HEN and GW have already been performed in the past with both ANTARES and ICECUBE neutrino telescopes [8, 9, 10]. In these proceedings are reported the results of the HEN follow-ups performed during the first Observation Run O1 of LIGO (September 2015-January 2016), i.e. the searches for neutrinos in temporal and spatial coincidence with GW150914, detailed in [11], and with GW151226 and the event candidate LVT151012, presented in [12]. Using GW data collected during O1, a so-called "sub-threshold" analysis is being performed, which searches for time and space correlations between HEN detected by ANTARES or ICECUBE and GW candidates of low signal-to-noise ratio; it is presented in the last section, with an emphasis on the specific procedure optimized to select HEN candidates in ANTARES data.

\section{HEN follow-ups of GW150914, GW151226 and LVT151012}

We searched for directional and temporal coincidences between the GW signals and reconstructed HEN candidates from both ANTARES and ICECUBE. Relying on the methodology defined in [13], we searched for $(i)$ temporal coincidences within a $\pm 500 \mathrm{~s}$ time window around the 
GW alert and (ii) spatial overlap between the $90 \%$ positional probability contour associated to each signal (see Fig. 1) and neutrino point spread function.

The search time window, which was used in previous GW+HEN searches, is a conservative, observation-based upper limit on the plausible emission of GW and HEN in the case of GRBs which are thought to be driven by a stellar-mass black hole-accretion disk system [14]. Directionally, we searched for overlap between the GW sky map (shown in Fig. 1) and the neutrino point spread functions, assumed to be Gaussian.
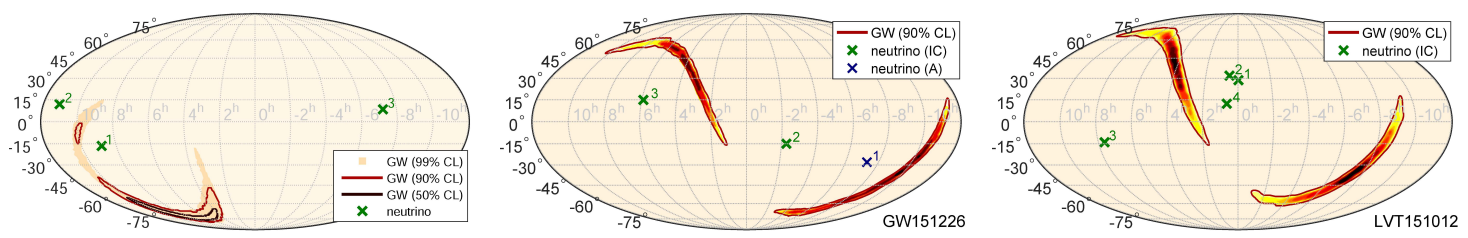

Figure 1: GW skymaps in equatorial coordinates, showing the reconstructed probability density contours of the GW events for GW150914 (left, [11]), GW151226 (middle, [12]), LVT151012 (right, [12]), and the reconstructed directions of high-energy neutrino candidates detected byICECUBE (green crosses) or ANTARES (blue crosses) during a $\pm 500 \mathrm{~s}$ time window around the $\mathrm{GW}$ events. The neutrino directional uncertainties are below $1^{\circ}$ and are not shown. GW shading indicates the reconstructed probability density of the GW events, darker regions corresponding to higher probability.

\subsection{HEN analysis for GW150914}

The ANTARES online reconstruction pipeline was used, which selects upgoing neutrino candidates with atmospheric muon contamination less than $10 \%$. An energy cut is also applied to reduce the background of atmospheric neutrinos which finally leads to an event rate of 1.2 events/day. Consequently, the expected number of neutrino candidates within 1000s is 0.014 , corresponding to a Poisson probability of observing at least one background event of $\sim 1.4 \%$. No neutrino candidates temporally coincident with GW150914 were found, which is fully consistent with the background expectation. The search for HEN counterpart with ICECUBE used the online event stream with an event selection similar to the one applied for point source searches, but optimized for real-time search. The expected background rate is equal to 2.2 atmospheric neutrino events in the Northern sky (atmospheric neutrinos) and 2.2 high energy atmospheric muons from the Southern sky. Within \pm 500 s around GW150914, three events were found, which are shown in Fig. 1 [11]. This observed event rate and the energy of these three events are compatible with the background expectations.

\subsection{HEN analysis for GW151226 and LVT151012}

We searched for coincident neutrinos within ANTARES data by selecting up-going events. The search was performed with the most recent official offline data set, incorporating dedicated calibrations, in terms of positioning, timing and efficiency. This sample is dominated by background events from mis-reconstructed down-going atmospheric muons. It was optimized for each GW event individually so that one event that passes the search criteria and is located within the $90 \% \mathrm{GW}$ probability contour would lead to a detection with a significance level of $3 \sigma$. For GW151226, a total of $1.4 \times 10^{-2}$ atmospheric neutrino candidates are expected in the field of view within $\pm 500 \mathrm{~s}$, 
while the number of misreconstructed down-going muons amounts to $8 \times 10^{-2}$ events in the same time window. We found one event that is temporally coincident with GW151226, located outside the $90 \%$ GW probability contour. The Poissonian probability of detecting at least one such background event when $9.4 \times 10^{-2}$ are expected is $\sim 9 \%$. Thus, this detection is consistent with the expected background muon rate and we conclude that this event is likely a misreconstructed downgoing muon. Its estimated deposited energy is $9 \mathrm{TeV}$, in agreement with what is expected from an atmospheric down-going muon. The sky location of the event is shown in Fig. 1 [12].

For LVT151210, the atmospheric neutrino candidate rate expected from the southern sky within $\pm 500 \mathrm{~s}$ is equal to $1.8 \times 10^{-2}$ while the number of misreconstructed down-going muons amounts to $4 \times 10^{-2}$. These are somewhat different from the values obtained for GW151226 as the sensitivity of ANTARES varies with time. No neutrino candidates temporally coincident with LVT151012 were found with ANTARES.

For ICECUBE, the detector online event stream was used. This event selection consists primarily of cosmic-ray-induced background events, with an expectation of 2.2 events in the northern sky (atmospheric neutrinos) and 2.2 events in the southern sky (high-energy atmospheric muons) per 1000 seconds. In the search window of \pm 500 s centered on the GW alert times, 2 and 4 neutrino candidates were found by IceCube in correspondence of GW151226 and LVT151012, respectively. This result is consistent with the expected background. The sky location of the neutrino candidates are shown in Fig. 1 [12].

\subsection{Constraints on neutrino emission}

We use the non-detection of joint GW and neutrino events to constrain neutrino emission from the GW source. Since the sensitivity of neutrino detectors is highly dependent on source direction, upper limits were calculated as a function of source direction for the whole sky. The obtained spectral fluence upper limits as a function of source direction are shown in Fig. 2, for a model with a spectral cutoff at high energies: $\left.d N / d E \propto E^{-2} \exp [-\sqrt{(} E / 100 \mathrm{TeV})\right]$. The latter model is expected for sources with exponential cutoff in the primary proton spectrum. A pure $E^{-2}$ was also considered and the results reported in [11, 12]. For a given direction, we adopt the upper limit from ICECUBE or ANTARES, whichever is more constraining.
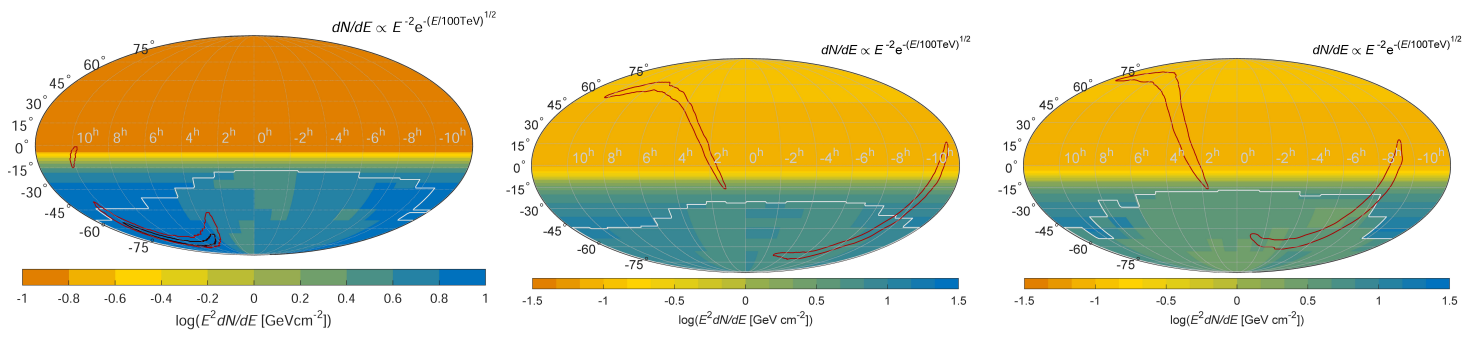

Figure 2: Upper limit on the high-energy neutrino spectral fluence $\left(v_{\mu}+\bar{v}_{\mu}\right)$ from GW150914 (left [11]), GW151226 (middle [12]), and LVT151012 (right [12]) as a function of source direction, assuming $d N / d E \propto$ $E^{-2} \exp [-\sqrt{(E / 100 \mathrm{TeV})}]$ neutrino spectrum. The region surrounded by a white line shows the part of the sky in which ANTARES is more sensitive (close to nadir). For comparison, the 50\% CL (for GW150914) and $90 \%$ CL contours (for the 3 events) of the GW sky maps are also shown. 
A constraint on the total energy emitted in neutrinos by the source can be obtained from the fluence upper limits, by integrating the emission within $[100 \mathrm{GeV}, 100 \mathrm{PeV}]$ for each considered source model. The obtained constraint will vary with respect to source direction, and also on the uncertain source distance. To account for these uncertainties for GW150914, we provide in Table 1 the range of energy values from the lowest to the highest possible within the $90 \%$ confidence intervals with respect to source direction and the $90 \%$ credible interval with respect to source distance (luminosity distance $D_{\mathrm{GW}}=410_{-180}^{+160} \mathrm{Mpc}$ ). For GW151226, the source position was constrained to within a 3D volume - the lower limit $D_{\text {low }}^{95 \%}(\vec{x})$ on the source distance for a given direction $\vec{x}$ such that the source is located within this distance at $95 \%$ credible level is used to calculate the upper limit on the total isotropic-equivalent energy emitted in neutrinos by the source, $\mathrm{E}_{v, \text { iso }}^{\mathrm{ul}}(\vec{x})=4 \pi\left[D_{\text {low }}^{95 \%}(\vec{x})\right]^{2} \int \frac{d N}{d E} E d E$. To quantify the range of the upper limits over the skymap, the minimum and maximum upper limit values over the whole GW skymap, separately for the two spectral models, are shown in Table 1. Constraints for LVT151012, not shown, are about a factor of 4 weaker as its expected distance is about twice that of GW151226 [2], while both their skymaps similarly lie over a large declination range, corresponding to similar neutrino detector sensitivities.

\begin{tabular}{c|c|c|c|c|c}
\hline GW Event & $D_{L}^{\mathrm{GW}}[\mathrm{Mpc}]$ & $\mathrm{E}_{\mathrm{GW}}^{\mathrm{iso}}[\mathrm{erg}]$ & $E^{-2}: \mathrm{E}_{\mathrm{HEN}}^{\text {iso }} / \mathrm{E}_{\mathrm{GW}}^{\text {iso }}$ & $E^{-2}$ with cutoff $: \mathrm{E}_{\mathrm{HEN}}^{\text {iso }} / \mathrm{E}_{\mathrm{GW}}^{\text {iso }}$ & Ref \\
\hline GW150914 & $410_{-180}^{+160}$ & $5 \times 10^{54}$ & $0.11 \%-26 \%$ & $0.13 \%-74 \%$ & {$[11]$} \\
\hline GW151226 & $440_{-190}^{+180}$ & $1.8 \times 10^{54}$ & $0.11 \%-17 \%$ & $0.17 \%-100 \%$ & {$[12]$} \\
\hline
\end{tabular}

Table 1: Minimum and maximum upper limits on the total energy radiated in neutrinos, for the pure $E^{-2}$ HEN spectrum, and the HEN spectrum with 100 TeV-cutoff, for GW150914 and GW151226 [11, 12].

\section{Searching for an association of weak GW signals and HEN candidates}

The GW signals for which we performed dedicated HEN followups were highly significant events (GW150914 and GW151226) or event candidates (LVT151012), with particularly high signal-to-noise ratios, shown in Fig. 3. Apart from these signals, LIGO search pipelines identified $\mathscr{O}(1000)$ potential signals, or triggers, characterized by low signal-to-noise ratios, as can be seen on Fig. 3. It is thus natural to search for a possible time and space correlation between these "sub-threshold" GW candidates (i.e., not significant enough to be claimed as "events") and HEN detected by ANTARES and ICECUBE, by performing a joint analysis, which method is presented in [14], and already applied for previous GW+HEN searches [10]. The joint analysis aims to identify GW events and HEN that originate from a common astrophysical source, by determining the significance of a GW+HEN event; this is obtained by a combination of the significances and directional distributions of GW events, neutrinos and galaxies (each galaxy is assigned a weight that reflects the probability of detecting an astrophysical HEN from the given galaxy, the distribution of GW sources being expected to be non-uniform in the volume probed by LIGO). The flow diagram of the joint GW+neutrino analysis algorithm is presented on Fig. 3, taken from [10]. Neutrinos detected by ANTARES and ICECUBE during O1 are used in this particular, still ongoing analysis. This section will concentrate on the specific process that has been developed for the selection of ANTARES HEN candidates. 

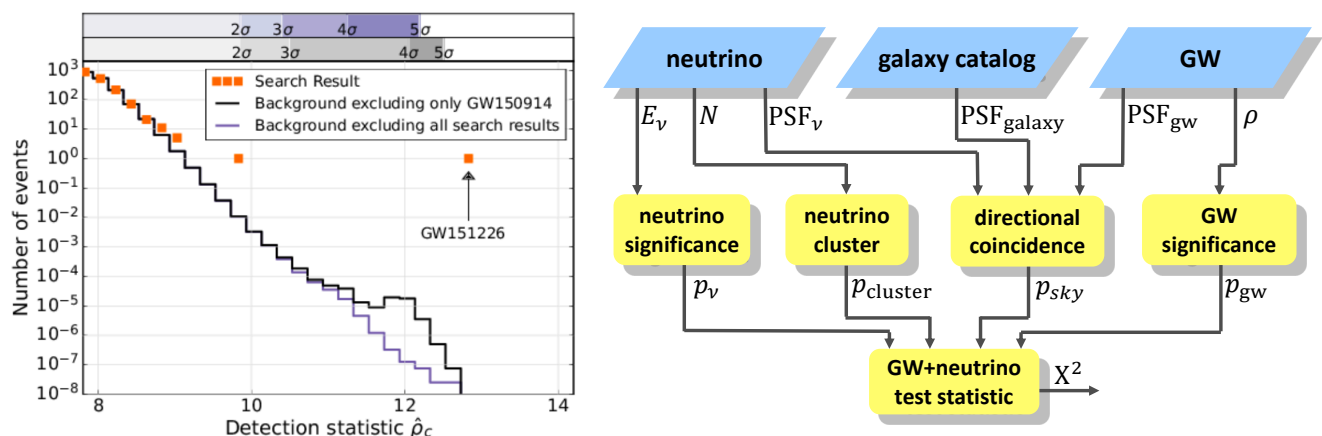

Figure 3: Left : As an illustration, GW detection statistics (related to the signal-to-noise ratio) distribution during O1, excluding GW150914, for the Binary Black-Hole search pipeline [15]. Right : Flow diagram of the joint GW+HEN analysis algorithm, showing how information on neutrinos, galaxies and GW are combined into one test statistic [10].

The selection of ANTARES $v_{\mu}$ candidates is data-driven : it is optimized in order to obtain a $3 \sigma$ significance for a HEN event in a sliding window of 1000s and within the expected reconstructed GW $90 \%$ probability contour for typical GW signals.

Time-dependent selection of the HEN candidates: In order to optimize the HEN search, we rely on [16] for a sample of realistic GW events, which allows to extract a relationship beteween the signal-to-noise ratio $\rho$ of a signal to the area of $90 \%$ confidence region $A_{90}$ for the GW localization, of the form $\log _{10} A_{90}=a \log \rho+b$, used to extrapolate the size of the confidence region to subtheshold GW events. This size is then convolved with ANTARES visible sky (ANTARES is only sensitive to up-going neutrinos in this analysis), and its local acceptance, to obtain the typical sizes reported in Table 2 below.

\begin{tabular}{c|c|c}
\hline $50 \%$ & $90 \%$ & $99 \%$ \\
\hline $524 \mathrm{deg}^{2}$ & $744 \mathrm{deg}^{2}$ & $1036 \mathrm{deg}^{2}$ \\
\hline
\end{tabular}

Table 2: $50 \%, 90 \%$ and $99 \%$ quantiles of the distribution of effective sizes (i.e. when the HEN detector effects, visibility and acceptance, are taken into account) of GW $90 \%$ probability regions.

A time-dependent selection criterium, based on the quality of the muon track reconstruction $\Lambda$, taking into account the time-dependence of the sensitivity of ANTARES, is then determined so that a selected HEN event in a time window of 1000 s, in a space angle corresponding to these typical GW-contour regions will yield a significance of $3 \sigma$ - the results for the time-dependent cut are shown in Fig. 4. For the final selection, the value corresponding to the median effective size has been used, which yields a total of 906 selected HEN candidates for ANTARES, for the period covering the entire O1 observation run from Sep 122015 to Jan 19 2016, corresponding to a rate of 8 neutrinos/day.

Characterization of HEN selected candidates: Each HEN candidate is characterized by its detection time, its arrival direction and reconstruction uncertainty, and a probability of being of atmospheric origin based on the number of detected photons $\mathrm{N}_{\text {hit }}$ (an estimator of the event deposited 
energy), shown in Fig. 4. At time of printing, the joint analysis, combining ANTARES and ICECuBE selected neutrinos, is still ongoing.e
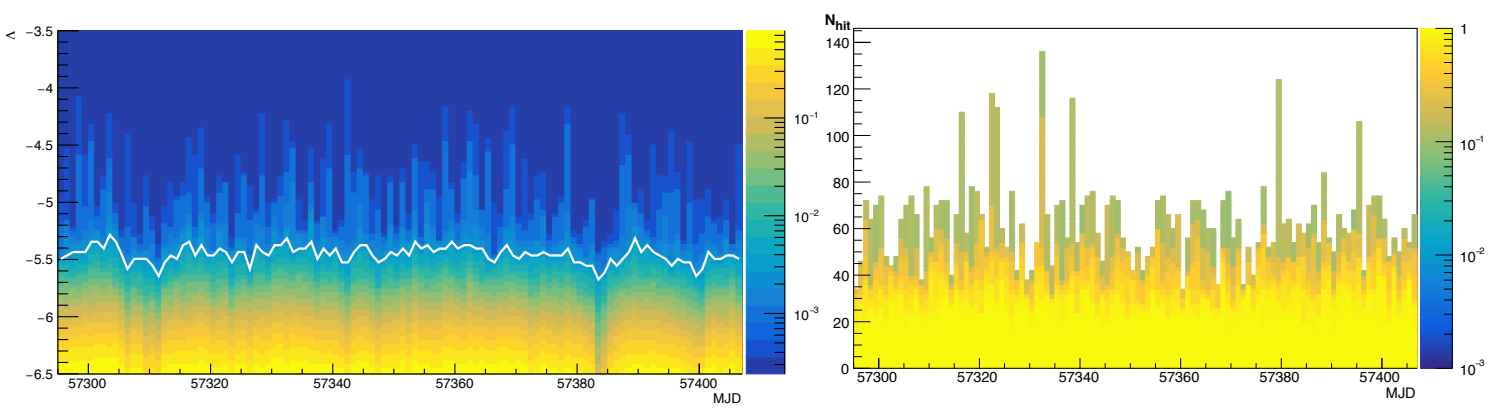

Figure 4: Left : probability to have $\geq 1$ background event with a value of the reconstruction parameter $\Lambda$ above a given value, in a 1000s time window, in a space area corresponding to the median of all $90 \% \mathrm{GW}$ contour regions. The white line marks the $3 \sigma$ significance level. Right : $p$-value of the HEN event being of atmospheric origin based on the data time-dependent distribution of the number of detected photons $\mathrm{N}_{\text {hit }}$.

Importance of a time-dependent analysis: Using a time-dependent selection criterium instead of a constant value as used in point-source searches [17] allows to increase the number of selected signal events, for a $E^{-2}$ spectrum, by $45 \% \pm 15 \%$, varying with time and data taking conditions. This optimization improves by a factor 1.5 to 2 the volume of universe probed by the joint analysis, and correspondingly the number of detectable joint GW+HEN sources.

\section{Conclusions \& Perspectives}

Searching in data recorded by the ANTARES Neutrino Telescope and ICECUBE concomittant with LIGO Observation Run O1, we detected no neutrino emission associated with the 2 confirmed signals GW150914 and GW151226, and event candidate LVT151012 [11, 12]. The total neutrino emission from the GW sources have been constrained, allowing for different possible neutrino spectra. HEN and/or electromagnetic emission induced by BBH systems can be expected in the case of significant gas accretion, which would trigger an energetic outflow. Such searches represent a promising way in comprehensively probing high-energy emission also for sources outside of the field of view of electromagnetic telescopes. Given the precise direction reconstruction of HEN $\left(\lesssim 1 \mathrm{deg}^{2}\right)$ compared to $\mathrm{GW}\left(\gtrsim 100 \mathrm{deg}^{2}\right)$, a joint detection would provide a greatly reduced sky area for follow-up observatories.

The principles of an analysis combining GW and HEN data have been presented, using lowsignificance GW signals and neutrinos detected by ANTARES and ICECUBE. The data-driven optimized procedure to select ANTARES neutrino candidates improves by a factor 1.5 to 2 the number of detectable joint GW+HEN sources. Such a search relies on the improved sensitivity of a combined GW+HEN search as compared to a single-messenger search, as already shown in [10].

Such follow-up searches and extended "sub-threshold" analyses will also be performed using data taken during the ongoing Observation Run O2 of LIGO, and soon VIRGO, with increased sensitivity, which has already led to the detection of GW170104 [3]. A dedicated HEN followup all-sky search for GW170104 using ANTARES data is presented in these Proceedings [18]. 


\section{References}

[1] Abbott B. P. et al. Phys. Rev. Lett. 116061102 (2016).

[2] Abbott B. P. et al. Phys. Rev. Lett. 116241103 (2016).

[3] Abbott B. P. et al. Phys. Rev. Lett. 118221101 (2017).

[4] e.g. Metzger B. D. and Berger E. Astroph. Journal 74648 (2012).

[5] e.g. Perna R., Lazzati D. and Giacomazzo B. Astroph. Journal Letters 82118 (2016).

[6] Heijboer A., on behalf of the ANTARES and KM3NET Collaborations, Highlights from ANTARES and KM3NET, Highlight talk, these Proceedings.

[7] Aartsen M. G. et al. Phys. Rev. Lett. 113101101 (2014).

[8] Adrìan-Martínez S. et al. JCAP 6008 (2013).

[9] Baret B., Procedings of the Rencontres de Moriond: Gravitation (2015).

[10] Aartsen M. G. et al. Phys. Rev. D 90102002 (2014).

[11] Adrìan-Martínez S. et al. Phys. Rev. D 93122010 (2016).

[12] Albert A. et al., https://arxiv.org/abs/1703.06298, accepted by Phys. Rev. D.

[13] Baret B. et al. Astropart. Phy. 35 1:7 (2011).

[14] Baret B. et al. Phys. Rev. D 85103004 (2012)

[15] Abbott B. P. et al. Phys. Rev. X 6041015 (2016)

[16] Singer L. P. et al. ApJ 795105 (2014)

[17] Adrìan-Martínez S. et al. Astroph. Journal 82365 (2016).

[18] Coleiro A., Dornic D., on behalf of the ANTARES Collaboration, Multi-messenger real-time analysis of transient events with the ANTARES neutrino telescope, presented as a Poster, these Proceedings. 